\title{
A Hybrid Dynamic-regenerative Damping Scheme for Energy Regeneration in Variable Impedance Actuators
}

\author{
Fan Wu and Matthew Howard*†
}

\begin{abstract}
Increasing research efforts have been made to improve the energy efficiency of variable impedance actuators (VIAs) through reduction of energy consumption. However, the harvesting of dissipated energy in such systems remains underexplored. This study proposes a novel variable damping module design enabling energy regeneration in VIAs by exploiting the regenerative braking effect of DC motors. The proposed damping module uses four switches to combine regenerative and dynamic braking, in a hybrid approach that enables energy regeneration without reduction in the range of damping achievable. Numerical simulations and a physical experiment are presented in which the proposed module shows an optimal trade-off between taskperformance and energy efficiency.
\end{abstract}

\section{INTRODUCTION}

Variable impedance actuators (VIAs) are believed to be the key for the next generation of robots to interact safely with uncertain environments and provide better performance in cyclic tasks and dynamical movements [1]. For example, the physical compliance incorporated in variable stiffness actuators (VSAs) (e.g., using elastic components such as springs) enables energy storage, which can (i) absorb external energy introduced into the system (e.g., from collisions) to enhance safety, and (ii) amplify output power by exploiting the stored energy [2].

Recently, much research effort has gone into the design of variable physical damping actuation, based on different principles of damping force generation (see [3] for a review). Variable physical damping has proven to be necessary to achieve better task performance when higher damping is desired to compensate undesired oscillations caused by the use of physical stiffness [4], [5]. It is also demonstrated in [4] that variable physical damping plays an important role in terms of energy efficiency for actuators with fixed stiffness or a small range of variable stiffness, to optimally adjust dynamic properties. However, while these studies represent important advances in terms of improving the efficiency of energy consumption in VIAs, the importance of variable physical damping may be underestimated, because the potential to harvest energy dissipated by damping has so far received little attention.

To address this, this paper extends the variable damping technique introduced by [6] to take into account of the energy regeneration capabilities of DC motors. In contrast to prior approaches that incorporate a power source directly (as done, for example, in [7]), a circuit design using four switchesconsidering the fact that the rotation of a revolute robot joint is bidirectional while typical power sources for energy storage are unidirectional-is introduced that enables adjustment of the electrical damping effect, while increasing the damping range available to the controller. The relation of the damping

*Fan Wu and Matthew Howard are with the Centre for Robotics Research, Department of Informatics, King's College London, UK $\{$ fan . wu, matthew.j.howard\} akcl.ac.uk.

${ }^{\dagger}$ This work was supported in part by the UK Engineering and Physical Sciences Research Council (EPSRC) SoftSkills project, EP/P010202/1.

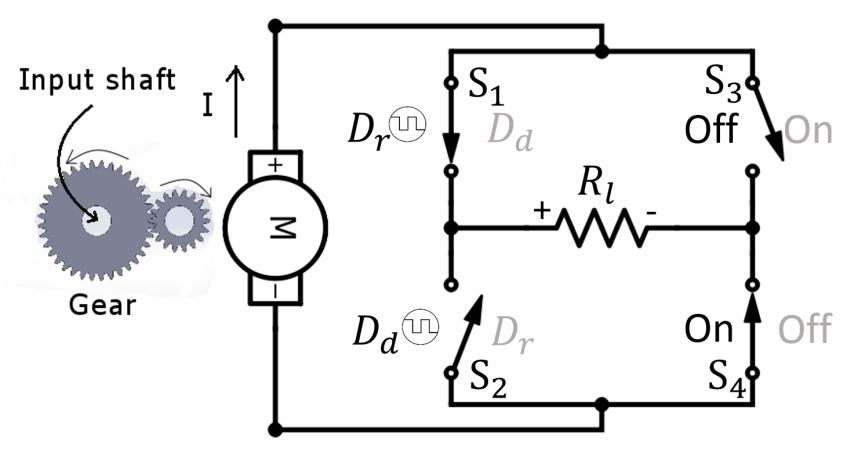

Fig. 1: Circuit diagram for bidirectional hybrid dynamicregenerative braking.

effect and the power of regeneration of the proposed damping module is investigated, and shows a non-monotonic relation emerges that requires balancing the trade-off between damping allocation and energy regeneration in a non-trivial way. Nevertheless, the proposed controllable damping module is evaluated in terms of movement performance and energy recovery of a simple reaching task on a ideal pendulum model and a more realistic VIA model, and shows that an optimal trade-off is achievable. Furthermore, an experiment is presented in which the damping module is realised in hardware, verifying the theoretical predictions about the damping module's behaviour.

\section{BACKGROUND}

To date, two main approaches have been proposed that enable variable damping through the use of DC motors, namely (i) dynamic braking and (ii) regenerative braking. In both cases, the back electromotive force is used to resist movement proportional to the effective resistance of the damper motor circuit, causing a variable damping effect. The following briefly outlines these schemes.

Scheme 11 - Dynamic braking: Dynamic braking in the context of VIA design was first proposed by [6]. A circuit diagram for this scheme is illustrated in Fig. 2|[a) In this mode, the damping effect is modulated by changing the duty-cycle $D_{d}$ that controls the portion of time that a switch $S_{1}$ spends in the open or closed position, thereby altering the effective resistance of the circuit. The damping coefficient follows the equation

$$
d=\frac{n_{d}^{2} k_{t}^{2} D_{d}}{R_{m}}=\bar{d}_{1} D_{d}
$$

where $n_{d}$ is the gear ratio of damping motor, $k_{t}$ is the torque constant and $R_{m}$ is the resistance of the motor. Note that, since $0 \leq D_{d} \leq 1$, the maximum damping coefficient that can be provided by dynamic braking is $\bar{d}_{1}=n_{d}^{2} k_{t}^{2} / R_{m}$.

In energy terms, dynamic braking is effective since it dissipates kinetic energy of the output link as heat in the electrical 

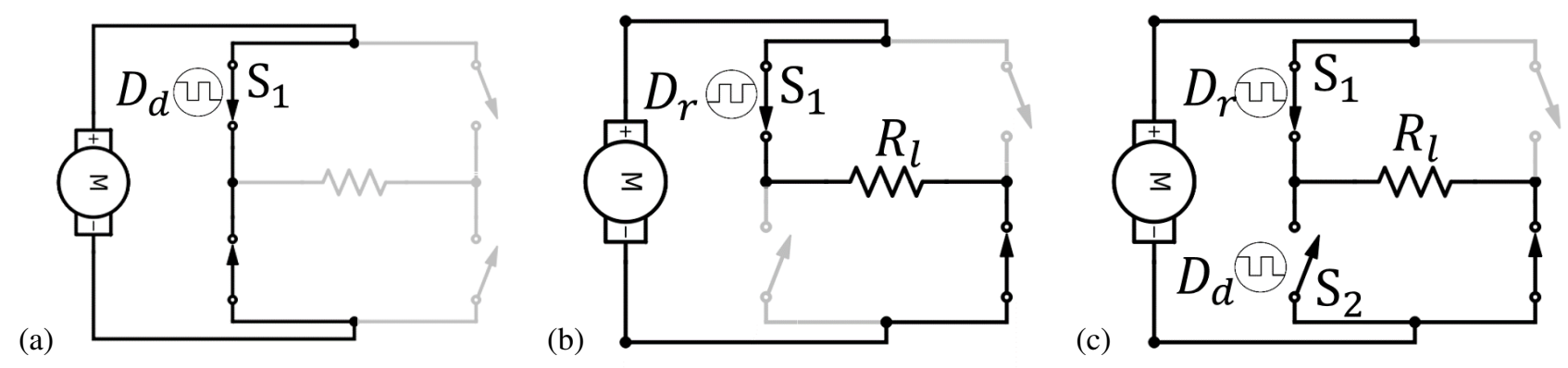

Fig. 2: Conceptual diagrams of (a) dynamic, (b) regenerative, and (c) hybrid dynamic-regenerative braking circuits.

circuit. It does not, however, charge energy to any electrical source, so the regeneration power is zero $\left(P_{\text {rege }}=0\right)$. In other words, this (potentially useful) energy is simply discarded, reducing the overall energy efficiency of the system.

Scheme 2 - Regenerative braking: Regenerative braking refers to the situation where the power generated by the motor through kinetic motion of the output link is used to recharge an electrical storage element (e.g., battery, supercapacitor). To implement regenerative braking, the electrical storage element can be simply connected to the circuit of the damping motor, as shown in [7]. In the context of VIA design, this can be implemented through the circuit in Fig. 2[(b).

In regenerative braking mode, the damping effect is dependent on the combined effective resistance of the circuit containing the electrical storage element. Similar to dynamic braking, this can be modulated by controlling the duty-cycle $D_{r}$ of a switch. The damping coefficient and the regeneration power can be calculated as

$$
\begin{aligned}
d & =\frac{n_{d}^{2} k_{t}^{2} D_{r}}{R_{m}+R_{l}}=\bar{d}_{2} D_{r} \\
P_{\text {rege }} & =\frac{R_{l} n_{d}^{2} k_{b}^{2} \dot{q}^{2} D_{r}}{\left(R_{m}+R_{l}\right)^{2}}=\alpha \bar{d}_{2} \dot{q}^{2} D_{r}
\end{aligned}
$$

respectively, where $R_{l}$ is the resistance of the electrical source and $\alpha=R_{l} /\left(R_{m}+R_{l}\right), k_{b}$ is back-EMF constant and equals to $k_{t}$.

Note that, introducing regenerative braking means that the mechanical energy that is otherwise discarded in the dynamic braking scheme can be harvested, enhancing the overall energy efficiency of the system. However, note also that, compared to dynamic braking, the maximum damping coefficient that can be produced by regenerative braking, $\bar{d}_{2}=n_{d}^{2} k_{t}^{2} /\left(R_{m}+R_{l}\right)$, is decreased since adding electrical load for charging increases the total equivalent resistance of the circuit. This can be a drawback in applications where higher levels of damping are needed (e.g., when there is need for a high dynamic response and therefore heavy braking of rapid movements).

\section{HYBRID DYNAMIC-REGENERATIVE BRAKING}

To address these issues, this paper proposes a variable damping scheme-termed hybrid braking - that switches between dynamic braking and pure regenerative braking to achieve the optimal benefits of both.

\section{A. Hybrid damping circuit}

The hybrid damping scheme is implemented through the circuit depicted in Fig. 2[(c) It uses two switches (denoted
$\left.S_{i}, i \in\{1,2\}\right)$ that switch at high frequency between (i) pure regenerative braking, and (ii) a blend of dynamic and regenerative braking. The principle by which the proposed scheme operates is as follows.

When switch $S_{2}$ is open, the module acts in regenerative braking mode, whereby current flows through the power storage element, with the effective resistance (damping level) determined by the duty cycle of $S_{1}$. (Note that, this results in an equivalent circuit to that used in Scheme 2, $c f$. Fig. 2[(b)]) On the other hand, when $S_{1}$ and $S_{2}$ are closed, there is a short circuit that causes current to bypass the resistive load $R_{l}$, creating a dynamic braking effect. In this case, the damping level can be determined by keeping $S_{1}$ closed and modulating the duty cycle of $S_{2}$. This enables a third braking scheme to be defined, alongside Schemes 1 and 2, as follows.

Scheme 3 - Hybrid braking: When the required damping $d^{*}$ is small enough, i.e., $d^{*} \leq \bar{d}_{2}$, it can be provided by pure regenerative braking, so $\bar{S}_{2}$ is opened $\left(D_{d}=0\right)$. When the required damping is greater, i.e., $d^{*}>\bar{d}_{2}, S_{1}$ is closed $\left(D_{r}=1\right)$ and $D_{d}$ is used to control $S_{2}$ to blend dynamic and regenerative braking.

The resulting damping coefficient and regeneration power are:

$$
\begin{aligned}
d & =\bar{d}_{2} D_{r}+\alpha \bar{d}_{3} D_{d} \\
P_{\text {rege }} & =\alpha \bar{d}_{2} \dot{q}^{2}\left(D_{r}-D_{d}\right) .
\end{aligned}
$$

Note that, if $D_{r}=D_{d}=1$, the same maximum damping coefficient as that achievable in a pure dynamic braking can be achieved, i.e., $\bar{d}_{3}=\bar{d}_{1}$. This, however, comes at the cost of the regeneration power vanishing $\left(P_{\text {rege }}=0\right)$.

\section{B. Hybrid Damping Control Modes}

In principle, each of the switches in the proposed circuit may be independently controlled by its own duty-cycle. While this enhances the flexibility of the damping module, it also introduces an undesirable layer of complexity to its control.

To address this, and enable the simple control of the module through a single control variable $u \in[0,1]$, the duty cycles of the switches can be coupled through the following relation

$$
\begin{aligned}
& D_{r}=\left\{\begin{array}{l}
\frac{u}{u_{r}}, u \leqslant u_{r} \\
1, u>u_{r}
\end{array}\right. \\
& D_{d}=\left\{\begin{array}{c}
0, u \leqslant u_{r} \\
\frac{u-u_{r}}{1-u_{r}}, u>u_{r}
\end{array}\right.
\end{aligned}
$$



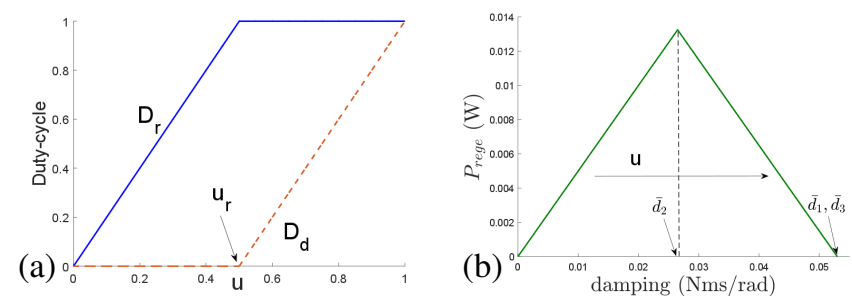

Fig. 3: Hybrid damping control modes. (a) Mapping from control input $u$ to duty cycles $D_{r}, D_{d}$. (b) Relation between regeneration power and damping.

where $u_{r}$ corresponds to the maximum of damping coefficient of regenerative braking $\left(d\left(u_{r}\right)=\bar{d}_{2}\right)$ and depends on user's selection. In this paper, $u_{r}$ is chosen to be 0.5 . Substituting (6) into (4), damping coefficient $d(u)$ as a function of $u$ is simplified to:

$$
d(u)=\bar{d}_{3} u
$$

As illustrated in Fig. 3(a), when $u \leqslant 0.5, D_{d}$ remains at zero (i.e., switch $S_{2}$ is open) and $D_{r}$ is linearly mapped from $u \in$ $[0,0.5]$ to $[0,1]$, while when $u>0.5, D_{r}$ is held at unity ( $D_{r}=1$ so $S_{1}$ is closed) and $D_{d}$ is linearly mapped from $u \in[0.5,1]$ to $[0,1]$.

The relation between the damping coefficient $d$ and the power regeneration $P_{\text {rege }}$ for a fixed angular velocity is shown in Fig. 3(b) As can be seen, the relationship is non-monotonic and there is a peak for $P_{\text {rege }}$ when $d=\vec{d}_{2}$, i.e., at the upper boundary of the pure regenerative braking domain.

\section{Bidirectional damping}

The hybrid damping circuit described so far enables the modulation of damping force associated with unidirectional motion of the output link. In order to realise damping of bidirectional motion (as is common in many robotic applications), it is necessary to ensure that the current generated by the damping motor always flows into the positive terminal of the electrical source. This can be achieved by a four-switch design of the damping circuit, as illustrated in Fig. 1. When the current flows from the positive terminal of the damping motor (as shown by the black arrow in Fig. 1,$S_{3}$ is open and $S_{4}$ is switched on. When the current flows from the negative terminal of the motor (as shown by the grey arrow), $S_{3}$ is closed and $S_{4}$ is open, and $S_{1}$ is controlled by $D_{d}$ and $S_{2}$ is controlled by $D_{r}$.

It should be further noted that, this latter circuit, implements the (bidirectional versions of) the two damping schemes outlined in $\mathrm{II}$ as special cases. For example, (i) holding $S_{2}, S_{3}$ open, $S_{4}$ closed and varying the duty cycle of $S_{1}$ results in regenerative braking, while (ii) holding $S_{3}, S_{4}$ open, $S_{1}$ closed, and varying the duty cycle of $S_{2}$ results in pure dynamic braking. In other words, the same hardware can be used to realise all three damping schemes.

\section{EVALUATION}

This section evaluates the proposed hybrid braking scheme in comparison to pure dynamic or regenerative braking through numerical simulation of (i) a simple pendulum actuated with an ideal VIA, and (ii) a more realistic simulation of a physical VIA, namely the Mechanically Adjustable Compliance and Controllable Equilibrium Position Actuator with Variable Damping (MACCEPA-VD) [6], [8].

\section{A. Simple pendulum with ideal VIA}

The aim of the first evaluation is to illustrate the effectiveness of the hybrid braking scheme in the context of a simple example task of target reaching.

For this, a model of a simple pendulum, subject to viscous friction and actuated by an ideal VIA 1 is used

$$
m l^{2} \ddot{q}+b \dot{q}=k\left(u_{2}\right)\left(u_{1}-q\right)-d\left(u_{3}\right) .
$$

Here, for simplicity, $m=1 \mathrm{~kg}, l=1 \mathrm{~m}, b=0.01 \mathrm{Nms} / \mathrm{rad}$. The right hand side of $(8)$ is the joint torque applied by the ideal VIA, $u_{1} \in[-\pi / 2, \pi / 2] \mathrm{rad}$ controls the equilibrium position and the stiffness $k\left(u_{2}\right)$ is proportional to the control input $u_{2} \in[0,1]$ :

$$
k\left(u_{2}\right)=\bar{k} u_{2}
$$

where $\bar{k}=200 \mathrm{Nm} / \mathrm{rad}$ is the maximum stiffness. The parameters ${ }^{2}$ that characterise the variable damping module are selected to be $\bar{d}_{3}=50 \mathrm{Nms} / \mathrm{rad}, \bar{d}_{2}=25 \mathrm{Nms} / \mathrm{rad}$ and $\alpha=0.5$. The control frequency is set to $50 \mathrm{~Hz}$.

The task is to reach a target $q^{*}=\pi / 3 \mathrm{rad}$ from the initial position $q=0 \mathrm{rad}$ within a finite time $t_{f}$ as quickly and accurately as possible, while minimising the energy consumption and control effort. This can be described through minimisation of the cost function

$$
\begin{aligned}
J=\int_{0}^{t_{f}}\left[w_{1}\left(q(t)-q^{*}\right)^{2}+\right. & w_{2}\left(u_{1}(t)-q^{*}\right)^{2} \\
& \left.+w_{3} u_{2}^{2}(t)-w_{4} P_{\text {rege }}\right] \mathrm{d} t
\end{aligned}
$$

where $w_{1}=1000, w_{2}=w_{3}=1, w_{4}=0.01$ are weighting parameters. These parameters are selected to take account of the different scales of the terms and allow reaching within a second.

To simplify the analysis, in the below, the command for equilibrium position is fixed at $u_{1}=\pi / 3$, while the commands for stiffness and damping are allowed to vary. The optimal open-loop control sequence for the latter is computed through the Iterative Linear Quadratic Regulator (ILQR) method [9] with the proposed hybrid braking scheme, and the resultant trajectory of the system is computed by simulating the execution of the open-loop command using the 4th Order RungeKutta method.

To evaluate the energy efficiency of the proposed approach, the total mechanical work and the total regenerated energy are computed from the resultant trajectories, i.e.,

$$
\begin{aligned}
E & =\int_{0}^{t_{f}} k\left(u_{1}-q\right) \cdot \dot{q} \mathrm{~d} t \\
E_{\text {rege }} & =\int_{0}^{t_{f}} P_{\text {rege }}(t) \mathrm{d} t,
\end{aligned}
$$

respectively. The net energy cost can be defined as

$$
E_{\text {net }}=E-E_{\text {rege }}
$$

\footnotetext{
${ }^{1}$ It is assumed that in the VIA model the damper is in parallel with the spring.

${ }^{2}$ These parameters are arbitrarily chosen to give response within a second. Experimentation shows the result is not sensitive to these values.
} 

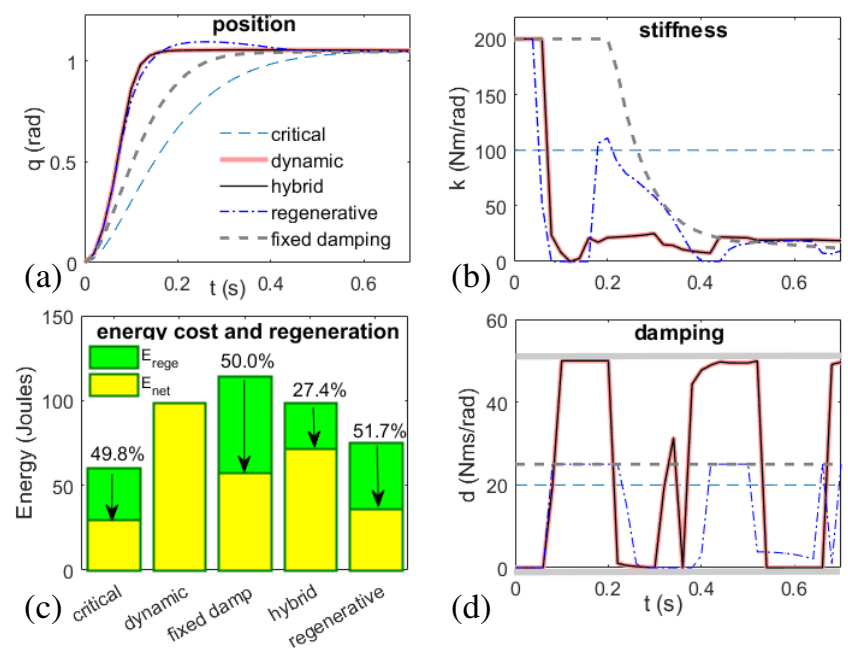

Fig. 4: Test of reaching task on simple pendulum with ideal VIA. Shown are optimal (a) joint angular trajectories, (b) stiffness, and (c) total mechanical work and percentage ratio of energy regeneration for different damping schemes, and (d) damping profiles.

The percentage ratio of energy regeneration 3 can be computed by

$$
\eta=\frac{E_{\text {rege }}}{E}
$$

For comparison, the experiment is repeated with (i) pure dynamic braking (Scheme 1), (ii) pure regenerative braking (Scheme 2), (iii) the case where the damping is fixed at the maximum power of regeneration $\left(d=\bar{d}_{2}\right)$, and (iv) a critically damped system. In the latter, the stiffness is chosen to be $k=100 \mathrm{Nm} / \mathrm{rad}$ and the damping is fixed to $d=20 \mathrm{Nms} / \mathrm{rad}$ such that the damping ratio $\zeta=d / 2 \sqrt{\mathrm{km}}=1$.

The results are illustrated in Fig. 4. As can be seen, the trajectory of the critically damped system reaches the target slowly but without overshoot (Fig. 4/a). The system with fixed damping reaches the target quicker than the critically damped one, because it can exploit the variable stiffness. The system with regenerative braking reaches the target quicker still, however, since the damping range is limited in this case, it suffers from overshoot once it reaches the target. In contrast, the dynamic braking and hybrid braking systems reach the target quickest without overshot, so perform best in terms of accuracy.

Looking at Fig. 4|(c), however, it can be observed that the dynamic braking performs worst in terms of net energy cost, since no energy is recovered throughout the movement. This contrasts with the hybrid approach, that achieves fast and accurate movement while also achieving $27.4 \%$ energy recovery, thereby lowering the net energy cost.

Overall, it appears that the proposed hybrid scheme offers the good trade-off between task accuracy and energy efficiency.

\footnotetext{
${ }^{3}$ Note that, for simplicity, it is assumed here that there is $100 \%$ kinetic to electric energy transmission efficiency of the DC motor. In practice, losses are likely to occur due to friction and losses in the conversion from the mechanical to the electrical domain.
}

VARIABLE IMPEDANCE ACTUATOR

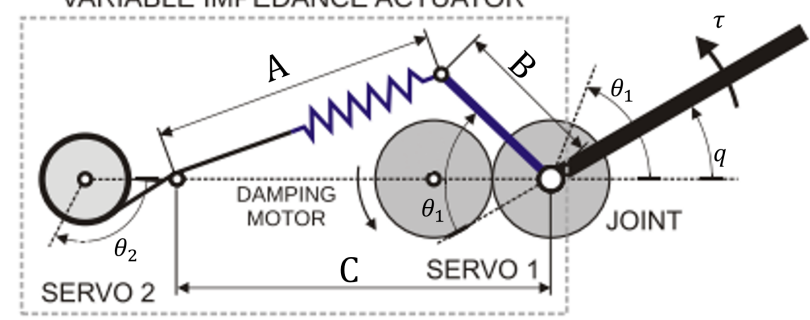

Fig. 5: Diagram of Mechanically Adjustable Compliance and Controllable Equilibrium Position Actuator [8] with variable damping [6]. In the results reported here, $B=3.6 \mathrm{~cm}, C=$ $13.5 \mathrm{~cm}, r=1.5 \mathrm{~cm}$ and the spring has linear spring constant $\kappa=394 \mathrm{~N} / \mathrm{m}$. The link has inertia $m=0.0015 \mathrm{kgm}^{2}$ and friction coefficient $b=0.0023 \mathrm{Nms} / \mathrm{rad}$.

\section{B. Optimal reaching with the MACCEPA-VD}

To evaluate the proposed scheme on a more realistic variable impedance actuation system, the MACCEPA-VD mechanism is chosen as an example. In the MACCEPA-VD, the equilibrium position and joint stiffness are controlled by two servomotors separately, while the damping coefficient is modulated by changing the duty cycle of the circuit of a DC motor attached rigidly to the joint. The system is illustrated in Fig. 5

The forward dynamics for this single joint system can be written as:

$$
\begin{aligned}
\ddot{q} & =\left(\tau_{s}-d\left(u_{3}\right) \dot{q}-b \dot{q}-\tau_{\text {ext }}\right) m^{-1} \\
\ddot{\theta}_{1} & =\beta^{2}\left(u_{1}-\theta_{1}\right)-2 \beta \dot{\theta}_{1} \\
\ddot{\theta}_{2} & =\beta^{2}\left(u_{2}-\theta_{2}\right)-2 \beta \dot{\theta}_{2}
\end{aligned}
$$

where $q, \dot{q}, \ddot{q}$ are the joint angle, velocity and acceleration, respectively, $b$ is the viscous friction coefficient for the joint, $m$ is the link inertia, $\tau_{s}$ is the torque generated by the spring force, and $\tau_{\text {ext }}$ is the joint torque due to external loading (the following reports results for the case of no external loading, i.e., $\tau_{\text {ext }}=0$ ). $\theta_{1}, \theta_{2}, \dot{\theta}_{1}, \dot{\theta}_{2}, \ddot{\theta}_{1}, \ddot{\theta}_{2}$ are the motor angles, velocities and accelerations. The motor angles $\theta_{1}, \theta_{2}$ are controlled by $u_{1} \in[-\pi / 3, \pi / 3], u_{2} \in[0, \pi / 3]$ respectively. The servomotor dynamics $(16),(17)$ are assumed to behave as a critically damped system, with $\beta=30$.

The torque $\tau_{s}$ can be calculated as follows:

$$
\tau_{s}=\kappa B C \sin \left(\theta_{1}-q\right)\left(1+\frac{r \theta_{2}-|C-B|}{A\left(q, \theta_{1}\right)}\right)
$$

where $A\left(q, \theta_{1}\right)=\sqrt{B^{2}+C^{2}-2 B C \cos \left(\theta_{1}-q\right)}, B$ and $C$ are the lengths shown in Fig. 5, $r$ is the radius of the winding drum used to adjust the spring pre-tension, and $\kappa$ is the linear spring constant.

The damping coefficient $d\left(u_{3}\right)$ depends on control input $u_{3}$ and is calculated according to the damping scheme used (i.e., (1), 2) or (7)). Note also that, the stiffness of this system depends on the joint and motor positions $q, \theta_{1}, \theta_{2}$

$$
\begin{aligned}
k\left(q, \theta_{1}, \theta_{2}\right) & =\kappa B C \cos \left(\theta_{1}-q\right)\left(1+\frac{r \theta_{2}-|C-B|}{A}\right) \\
& -\frac{\kappa B^{2} C^{2} \sin ^{2}\left(\theta_{1}-q\left(r \theta_{2}-|C-B|\right)\right)}{A^{\frac{3}{2}}} .
\end{aligned}
$$



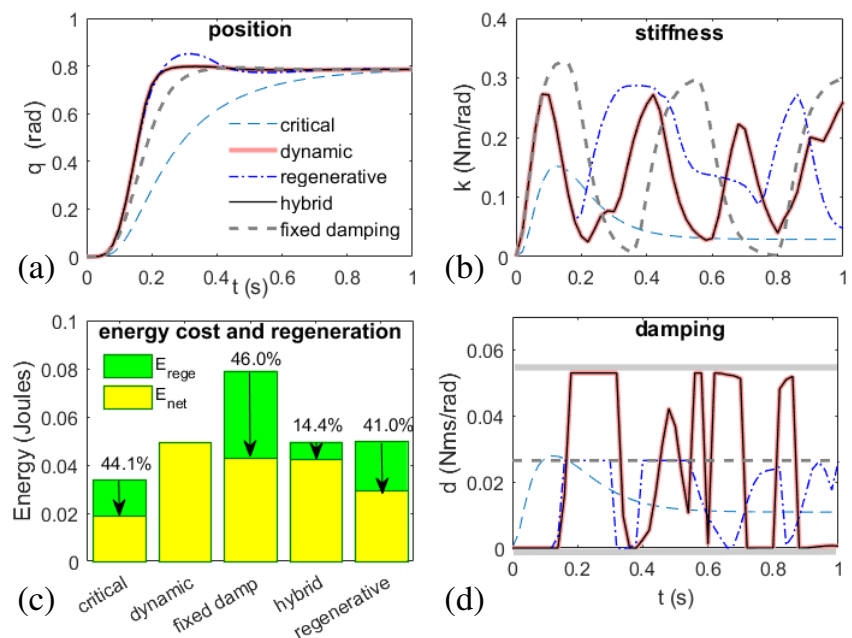

Fig. 6: Test of reaching task with the MACCEPA-VD. Shown are optimal (a) joint angular trajectories, (b) stiffness, and (c) total mechanical work and percentage ratio of energy regeneration for different damping schemes, and (d) damping profiles.

To evaluate the proposed hybrid damping method, ILQR is used to determine the optimal open-loop control sequence for the task of reaching a target point $q=\pi / 4 \mathrm{rad}$ starting from initial state ${ }^{4} \mathbf{x}_{0}=(0,0,0,0,0,0)^{\top}$ within finite time $t_{f}=2 \mathrm{~s}$, using the proposed hybrid damping scheme (see $\$$ III). The cost function for optimisation takes the same form as (10), where weighting parameters are $w_{1}=10^{3}, w_{2}=w_{3}=10^{-4}, w_{4}=$ $10^{-6}$.

For comparison, the experiment is repeated using dynamic and regenerative braking (Schemes 1 and 2), a fixed damping coefficient of $d=\bar{d}_{1}$, and a 'critically damped' system in which, following [6], the instantaneous damping ratio is held at $\zeta(t)=1$ by enforcing the stiffness-damping relationship $d(t)=2 \sqrt{k(t) m}$.

The results are shown in Fig. 6. There, it can be seen that, the 'critically damped' system avoids overshoot, but reaches the target slowly. It also has the lowest energy consumption, in part due to its sluggish response in moving to the target. The fixed damping trajectory reaches the target faster, but slower than the trajectories using dynamic and hybrid schemes. Although it regenerates the most energy, it also incurs the highest cost in terms of mechanical energy, so overall the net energy cost is higher than Schemes 2 and 3 (see Fig. 6(c)).

The regenerative braking scheme has better energy efficiency as its net energy cost is lower but it suffers greatest overshoot due to its restricted damping range, while the dynamic braking scheme achieves higher accuracy but at the expense of higher net energy cost. The former has higher total mechanical work, but regenerates $41.0 \%$ and results in lower net energy cost. In comparison, hybrid braking achieves almost identical performance in terms of accuracy, but at higher energy efficiency.

\section{EXPERIMENT}

This section presents an experiment using a physical device to

${ }^{4}$ Following [6], the state vector is defined as $\mathbf{x}=\left(q, \dot{q}, \theta_{1}, \theta_{2}, \dot{\theta}_{1}, \dot{\theta}_{2}\right)^{\top}$.

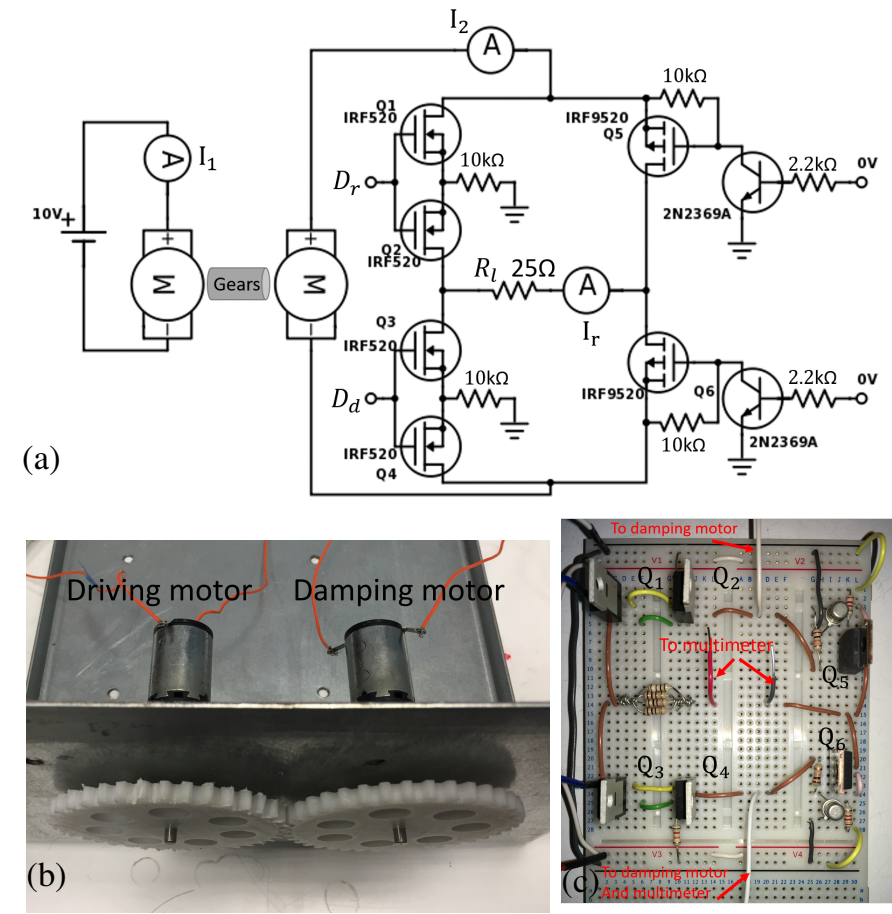

Fig. 7: Damping and power regeneration measurement experiment setup. Shown are (a) circuit diagram of the experiment setup, (b) damping test rig, and (c) damping circuit module.

verify the design of the hybrid damping circuit and its control scheme.

The experimental set up is shown in Fig. 7 As a simple test-rig, two identical DC motors (Maxon A-max 22/110125) are coupled through a pair of spur gears to enable one motor (driver) to drive the other (damper), see Fig. 7] b) The two motors have the same gearhead with $n_{d}=20$. The torque constant is $k_{t}=0.0212 \mathrm{Nm} / \mathrm{A}$ and the motor resistance $R_{m}=$ $21.2 \Omega$.

The damper motor is connected to the circuit depicted in Fig. 7||a), that is the physical realisation of the conceptual diagram Fig. 1. In this circuit design, a pair of N-channel MOSFETs (IRF520) is used as one switch to make sure that the switching mechanism works properly for bidirectional current. In Fig. 7| (1) the pair of $Q_{1}, Q_{2}$ works as the switch $S_{1}$, and $Q_{3}, Q_{4}$ make the switch $S_{2}$. Two P-channel MOSFETs (IRF9520) with BJTs (2N2369A) are used as switches $S_{3}, S_{4}$. The duty-cycles $D_{r}, D_{d}$ are controlled by PWM signals from an Arduino Mega2560 board. By setting $0 V$ signals on the control pins for $Q_{5}, Q_{6}$, they are open for just one current direction but closed for the other. For the ease of power measurement, a resistor is used to represent the electrical load $\left(R_{l}=25 \Omega\right)$.

In the experiment, the driving motor is used to drive the system while the damping applied by the second motor is varied, and the resultant motion (motor speeds and energy regeneration) is recorded. Specifically, the driving motor is powered by a $10 \mathrm{~V}$ DC power supply $\left(V_{b b}=10 \mathrm{~V}\right)$ while the damping motor control input $u$ is varied from 0 to 1 in increments of 0.1 (with the corresponding duty-cycles $D_{r}, D_{d}$ computed by (6)). Simultaneously, three multimeters (Rapid DMM 318) are used to measure the currents $I_{1}, I_{2}, I_{r}$ through the driving motor, damping motor and the electrical load $R_{l}$ 


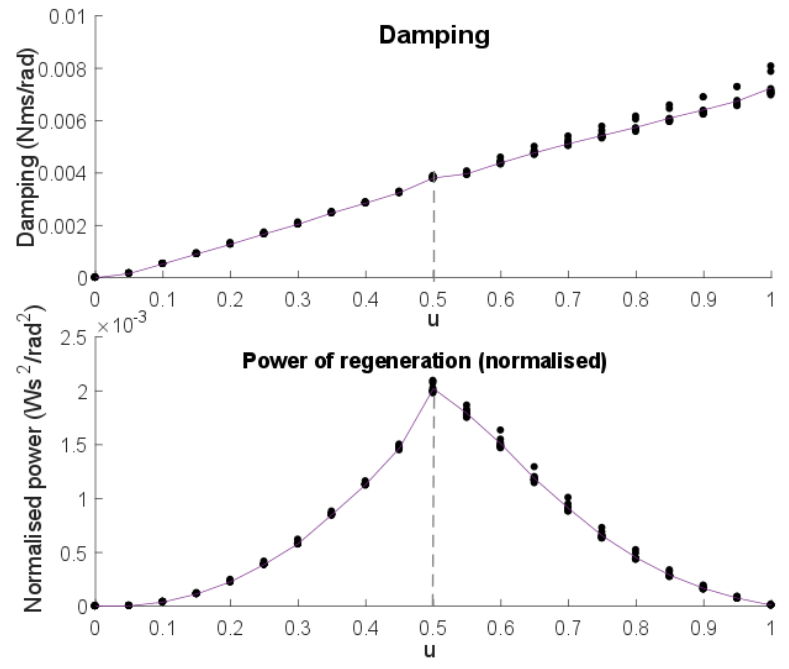

Fig. 8: Results of the damping test experiment. The damping coefficient (top) and regeneration power (bottom) for each tested control input $u \in[0,1]$ are shown. Black dots represent the data points for 10 repetitions of the experiment and the curves show the mean values.

respectively. The latter data are used to compute the angular speed of the motors $\omega$ and the damping torque $\tau_{d}$ according to

$$
\begin{aligned}
V_{b b} & =I_{1} R_{m}+n_{d} k_{t} \omega \\
\tau_{d} & =n_{d} k_{t} I_{2}=d(u) \omega
\end{aligned}
$$

The damping coefficient $d(u)$ for a given $u$ is then

$$
d(u)=\frac{n_{d}^{2} k_{t}^{2} I_{2}}{V_{b b}-I_{1} R_{m}}
$$

and the regeneration power (normalised by the square of speed for comparison) is estimated as

$$
P_{\text {rege }}=\frac{n_{d}^{2} k_{t}^{2} I_{r}^{2} R_{l}}{\left(V_{b b}-I_{1} R_{m}\right)^{2}} .
$$

The results based on the data collected from 10 repetitions of the experiment is plotted in Fig. 8 .

There it can be seen that, the experimental data is in good agreement with the theoretical predictions (see increasing $u$ from 0 to 1 , the damping coefficient $d$ increases almost proportionally. Furthermore, it is verified that, when fixing the angular speed ( $P_{\text {rege }}$ has been normalised), the relation between $P_{\text {rege }}$ and $u$ is non-monotonic with a peak found at $u=0.5$.

\section{Conclusions}

This paper proposes an extension to variable damping module design for VIAs based on the motor braking effect. In contrast to previous, pure dynamic braking designs, the proposed approach provides a solution for realising controllable damping, which enables the VIA to regenerate dissipated energy from bidirectional rotation movement to charge a unidirectional electric storage element. Furthermore, it overcomes the drawback of a reduction in the maximum damping effect found in pure regenerative braking schemes.
The control input for this damping module simply varies from 0 to 1 , representing a proportional percentage of the maximum damping. As the power regeneration has a nonmonotonic relation with the control input and damping coefficient (as verified by experiment), the balancing between damping allocation and energy regeneration needs to be treated with care. However, application of the hybrid damping module to VIAs in simulation, shows that it offers more flexibility to balance the trade-off between task performance and energy cost.

In future work, it is intended to investigate the role of variable damping and energy regeneration by considering more use cases and more performance criterion such as reaching time, stability and robustness. Analysis of transmission efficiency of the regenerative damping module will be conducted, by taking account of more factors causing losses during crossdomain energy conversion. Furthermore, it is planned to realise a hardware implementation that integrates the hybrid damping module into a sensorised VIA to measure the combined energy consumption and regeneration during operation. Such information can be used for optimal decision making for task performance and energy cost trade-off.

\section{REFERENCES}

[1] B. Vanderborght, A. Albu-Schaeffer, A. Bicchi, E. Burdet, D. Caldwell, R. Carloni, M. Catalano, G. Ganesh, M. Garabini, M. Grebenstein, G. Grioli, S. Haddadin, A. Jafari, M. Laffranchi, D. Lefeber, F. Petit, S. Stramigioli, N. Tsagarakis, M. Van Damme, R. Van Ham, L. C. Visser, and S. Wolf, "Variable impedance actuators: Moving the robots of tomorrow," in IEEE Int. Conf. Intelligent Robots \& Systems, 2012.

[2] M. Grebenstein, A. Albu-Schaffer, T. Bahls, M. Chalon, O. Eiberger, W. Friedl, R. Gruber, S. Haddadin, U. Hagn, R. Haslinger, H. Hoppner, S. Jorg, M. Nickl, A. Nothhelfer, F. Petit, J. Reill, N. Seitz, T. Wimbock, S. Wolf, T. Wusthoff, and G. Hirzinger, "The DLR hand arm system," in IEEE Int. Conf. Robotics \& Automation, 2011.

[3] B. Vanderborght, A. Albu-Schäffer, A. Bicchi, A. Albu-Schaeffer, A. Bicchi, E. Burdet, D. Caldwell, R. Carloni, M. Catalano, O. Eiberger, W. Friedl, G. Ganesh, M. Garabini, M. Grebenstein, G. Grioli, S. Haddadin, H. Hoppner, A. Jafari, M. Laffranchi, D. Lefeber, F. Petit, S. Stramigioli, N. Tsagarakis, M. Van Damme, R. Van Ham, L. Visser, and S. Wolf, "Variable impedance actuators: A review," in IEEE Int. Conf. Intelligent Robots \& Systems, 2013.

[4] M. Laffranchi, L. Chen, N. G. Tsagarakis, and D. G. Caldwell, "The role of physical damping in compliant actuation systems," in IEEE Int. Conf. Intelligent Robots \& Systems, 2012.

[5] M. Laffranchi, N. G. Tsagarakis, and D. G. Caldwell, "CompAct Arm: a Compliant Manipulator with Intrinsic Variable Physical Damping," in Robotics: Science and Systems VIII, 2013.

[6] A. Radulescu, M. Howard, D. J. Braun, and S. Vijayakumar, "Exploiting variable physical damping in rapid movement tasks," in IEEE/ASME Int. Conf. Advanced Intelligent Mechatronics, 2012.

[7] J. M. Donelan, Q. Li, V. Naing, J. A. Hoffer, D. J. Weber, and A. D. Kuo, "Biomechanical Energy Harvesting: Generating Electricity During Walking with Minimal User Effort," Science, no. 5864, pp. 807-810, 2008.

[8] R. Van Ham, B. Vanderborght, M. Van Damme, B. Verrelst, and D. Lefeber, "MACCEPA, the mechanically adjustable compliance and controllable equilibrium position actuator: Design and implementation in a biped robot," Rob. Auton. Syst., vol. 55, no. 10, pp. 761-768, 2007.

[9] W. Li and E. Todorov, "Iterative Linear Quadratic Regulator Design for Nonlinear Biological Movement Systems," in IEEE Int. Conf. Robotics \& Automation, 2004 